archives-ouvertes

\title{
REM-Based Handover Algorithm for Next-Generation Multi-Tier Cellular Networks
}

\author{
Cristo Suarez-Rodriguez, B Jayawickrama, Faouzi Bader, E Dutkiewicz, \\ Michael Heimlich
}

\section{To cite this version:}

Cristo Suarez-Rodriguez, B Jayawickrama, Faouzi Bader, E Dutkiewicz, Michael Heimlich. REMBased Handover Algorithm for Next-Generation Multi-Tier Cellular Networks. IEEE Wireless Communications and Networking Conference (IEEE WCNC'2018), Apr 2018, Barcelone, Spain. 10.1109/wcnc.2018.8377242 . hal-01705711

\section{HAL Id: hal-01705711 https://hal.archives-ouvertes.fr/hal-01705711}

Submitted on 9 Feb 2018

HAL is a multi-disciplinary open access archive for the deposit and dissemination of scientific research documents, whether they are published or not. The documents may come from teaching and research institutions in France or abroad, or from public or private research centers.
L'archive ouverte pluridisciplinaire HAL, est destinée au dépôt et à la diffusion de documents scientifiques de niveau recherche, publiés ou non, émanant des établissements d'enseignement et de recherche français ou étrangers, des laboratoires publics ou privés. 


\title{
REM-Based Handover Algorithm for Next-Generation Multi-Tier Cellular Networks
}

\author{
Cristo Suarez-Rodriguez ${ }^{* \ddagger}$, B. A. Jayawickrama ${ }^{\dagger}$, Faouzi Bader ${ }^{\ddagger}$, E. Dutkiewicz ${ }^{* \dagger}$ and Michael Heimlich ${ }^{* \dagger}$ \\ ${ }^{*}$ School of Engineering, Macquarie University, Sydney, Australia \\ Email: cristo.suarez-rodriguez@hdr.mq.edu.au, michael.heimlich@mq.edu.au \\ ${ }^{\dagger}$ University of Technology Sydney, Global Big Data Technologies Centre, Sydney, Australia \\ Email: \{beeshanga.jayawickrama, eryk.dutkiewicz\}@uts.edu.au \\ ${ }^{\ddagger}$ SCEE/IETR, CentraleSupélec, Rennes, France \\ Email: faouzi.bader@supelec.fr
}

\begin{abstract}
The strongest-cell criterion has been extensively used for handover algorithms during the last cellular-network generations. When network topologies become multi-layered, it results in abrupt behaviors such as the ping-pong effect as a consequence of the power gap between tiers and their irregular deployment. This effect not only affects users' quality of experience but also introduces a significant network overhead. Therefore, we propose an original handover algorithm based on predicted incomplete channel states from a Radio Environment Map to reduce this effect. The proposed algorithm is user triggered, network assisted, and fully backward compatible with LTE-A. Moreover, we evaluate the performance of our proposed algorithm against LTE-A in a two-tier cellular network for different user speeds following the guidelines outlined by the 3GPP on diverse matters (channel, mobility, wrapping, etc.). When applying realistic timing, our results reveal a highly substantial improvement in the number of ping-pong handovers regardless of the handover policy adopted in comparison to LTEA without sacrificing users' experience; for instance, we obtain at least an order of magnitude decrease in the ping-pong rate at the expense of losing less than 9 percent in spectral efficiency.

Index Terms-Radio Environment Maps, Handover, Heterogeneous Networks.
\end{abstract}

\section{INTRODUCTION}

As a result of the rapidly growing demands of multimedia data-intensive applications such as video streaming, gaming, social networking, etc., current cellular networks, e.g. Long Term Evolution (LTE) and LTE-Advanced (LTE-A), cannot satisfy users' needs any more [1]. This has led the $3^{\text {rd }}$ Generation Partnership Project (3GPP) to design LTE-A Pro, which is meant to pave the way towards a seamless transition to $5 \mathrm{G}$. Among others, $5 \mathrm{G}$ is expected to enhance broadband connectivity by using LTE in the unlicensed spectrum below $6 \mathrm{GHz}$. However, it simultaneously raises a twofold issue: on the one hand, a large portion of the spectrum becomes available for carrier aggregation; however, on the other hand, coexistence across different technologies can also bring higher levels of interference. This represents an opportunity to expand the Heterogeneous Network (HetNet) network layout to a greater extent, combining not only licensed spectrum, as usual, but also unlicensed spectrum to create a multi-tier cellular network.
The ultimate motivation behind the deployment of small cells (micro, nano, pico, femto) underlying the coverage of macrocells is improving the capacity per unit area. Despite its countless advantages with respect to the perceived Quality of Service (QoS) from the users' perspective, Mobile Network Operators (MNO) must overcome a set of challenges like efficient network management or uninterrupted mobility. Traditionally, the LTE handover (HO) algorithm has proven to perform quite well in macro-only scenarios, however it struggles in the presence of small cells due to their non-collocated layout and low transmit power [2]. A comprehensive survey of handover decision algorithms for femtocells in LTE-A is delivered in [3]. However, to the best of our knowledge, it is rare to find research works comparing them to decide on which one to consider under certain circumstances.

We introduce the idea of making use of Radio Environment Maps (REM) as a powerful tool for a handover decision algorithm. A REM is basically a heat map of the RSS in $\mathbb{R}^{2}$ for a specific band. In particular, we are interested in improving mobility performance by reducing the overall number of handovers without sacrificing network capacity. In a multi-tier scenario, this means reducing the number of redundant handovers, also known as ping-pong handovers, between tiers, especially for high-speed User Equipments (UE). Ping-pong handovers are defined as consecutive handovers between the same cells within a short period of time, e.g. less than a second. REMs could potentially address simultaneously a myriad of users ranging from pedestrians to trains. Our proposed algorithm aims at camping users, not only on the strongest cell available but also on the one with the highest Signal-to-Interference-plus-Noise Ratio (SINR) based on the current user's trajectory.

Section II presents an overview of the system model employed. Section III explains the REM-based handover algorithm. Section IV provides numerical performance results of the proposed algorithm benchmarked against LTE-A. Finally, Section V states the main findings of this work as well as a summary. 


\section{SYSTEM MODEL}

In this work, we assume that REMs are stored and managed by the mobile network operator.For example, in [4] the authors discuss possible implementations in different network entities within an LTE-A network. They introduce a layered architecture that consists of local and global REMs, where the coordination between local REMs can be done through the $\mathrm{X} 2$ interface while the coordination between local and global REMs must be done through the S1 one. REMs are essentially geolocation databases that store some Radio-Frequency (RF) relevant parameters. The cost, field measurements mostly, of the maps is not considered in this work. So as to create a map, there are basically two options that can be followed: we can deploy measurement-capable devices that perform measurements (UEs and sensors) and apply spectrum cartography techniques as in [5], or we can use a realistic 3D urban electromagnetic wave propagation fused with digital geographical map data (terrain altitude, building contours, etc.) to synthesize coverage maps (up to 5-m resolution) as detailed in [6].

In addition, users must be able to measure the band of interest and report those measurements to their serving cell in a similar fashion to the current LTE-A UE. Moreover, we need UEs to have the capability of positioning using either a Global Navigation Satellite System or an LTE-A networkassisted technique. In the former group is included the GPS, GLONASS or GALILEO positioning systems, whereas in the latter Observed Time-Difference Of Arrival (OTDOA) and Enhanced Cell-ID techniques are used. The impact of these systems on the proposed algorithm is beyond the scope of this work.

We assume a Heterogeneous Network (HetNet) composed of evolved Node B (eNB) and Home eNB (HeNB) in openaccess mode. Both tiers belong to the same MNO, whose allocated frequency bands compose the set $\mathcal{F}$. Additionally, they operate in the same band $f \in \mathcal{F}$. As we evaluate the handover performance, from now on, we are solely focused on the downlink. Let $\mathcal{C}$ denote the set of cells operating in $f$, regardless of the tier, and $\mathcal{U}$ the set of users. In consequence, the SINR of a user $u \in \mathcal{U}$ camping on its serving cell $s \in \mathcal{C}$ can be expressed as

$$
\gamma_{s, u}=\frac{P_{s}\left|h_{s, u}\right|^{2}}{\sum_{c \in \mathcal{C} \backslash\{s\}} P_{c}\left|h_{c, u}\right|^{2}+\sigma_{u}^{2}}
$$

where $P_{s}$ is the transmit power of the cell $s, P_{c}$ is the transmit power of the cell $c, h_{s, u}$ is the channel gain from cell $s$ to user $u, h_{c, u}$ is the channel gain from cell $c$ to user $u$, and $\sigma_{u}^{2}$ is the noise power at user $u$. Nonetheless, the LTE-A standard defines two different UE measurements: Reference Signal Received Power (RSRP) and Reference Signal Received Quality (RSRQ). Without loss of generality, in our approach a user $u$ only measures the RSS from a cell $c \in \mathcal{C}$, defined as $\operatorname{RSS}_{c, u}=P_{c}\left|h_{c, u}\right|^{2}$.

LTE-A caters for mobility with event-triggered reporting, using both intra- and inter-frequency measurements whose values and thresholds are in $\mathrm{dBm} / \mathrm{dB}$. The entry condition must be met for at least the Time To Trigger (TTT) period, generally configured by the network and further scaled by the user, depending on its speed to control the ping-pong effect. A relative offset also helps to adjust the timing when the handover is executed: a low value might lead to a tooearly handover while a high value may end up with a too-late handover or even a Radio Link Failure (RLF). Substituting the RSRP for the RSS as aforementioned we can describe this handover policy as follows:

$$
c^{*}=\underset{c \in \mathcal{C}}{\operatorname{argmax}} \operatorname{RSS}_{c, u}(t) \mid \operatorname{RSS}_{s, u}^{\mathrm{TTT}}<\mathrm{RSS}_{c, u}^{\mathrm{TTT}}-\mathrm{HHM}
$$

where $c^{*}$ corresponds to the strongest cell, $\operatorname{RSS}_{c, u}^{\mathrm{TTT}}$ is the $\mathrm{RSS}_{c, u}$ during the TTT, and HHM stands for Hysteresis Handover Margin and corresponds to the relative offset. We would like to emphasize the time dependence, which is not normally made explicit since our proposed algorithm to be introduced in the next section will depend specifically on time. From (2) it is clear that the current algorithm promotes cells with higher power/channel gain regardless of any other criteria such as SINR, cell load, UE power consumption, etc. In the remainder of this work, cell detection is assumed to be done under the time constraints specified in 3GPP TS 36.133 [7], therefore we will only focus on the handover itself.

\section{REM HANDOVER Algorithm Description}

While the LTE-A handover policy has shown itself to be robust enough only in macrocell scenarios, with HetNets it becomes more challenging due to the higher interference under co-channel deployment, Neighbor Cell Lists (NCL) changing rapidly when femtocells appear and disappear, etc. This is especially true for users who pass through an area under the overlapped coverage of different tier cells, because the number of ping-pong handovers increases and, thus, the network signaling. Furthermore, the higher the UE speed is, the more complicated the handover decision stage becomes. Our main objective is to reduce the overall number of pingpong handovers between tiers using the spatial information provided by REMs without compromising the QoS of the users.

The proposed algorithm scheme relies on the same eventtriggered mechanism as does LTE-A, although it is not limited to it. Thus it can be seamlessly adapted to current state-of-theart UEs. As REMs will be only stored on the network side, the proposed algorithm avoids any extra overhead due to the exchange of information between the REM database and the UE. However, users must be requested to send their location along with the measurement reports, as already happens in the Minimization of Drive Test (MDT) in LTE-A [8].

Once the measurement report is triggered, our proposed algorithm predicts the user's position in a timespan $\Delta t$, assuming a simple uniform linear motion described by $\mathbf{r}(t+$ $\Delta t)=\mathbf{r}(t)+\mathbf{v} \Delta t$, where $\mathbf{r}$ and $\mathbf{v}$ are position and velocity respectively. Even if the trajectory of the user is either 


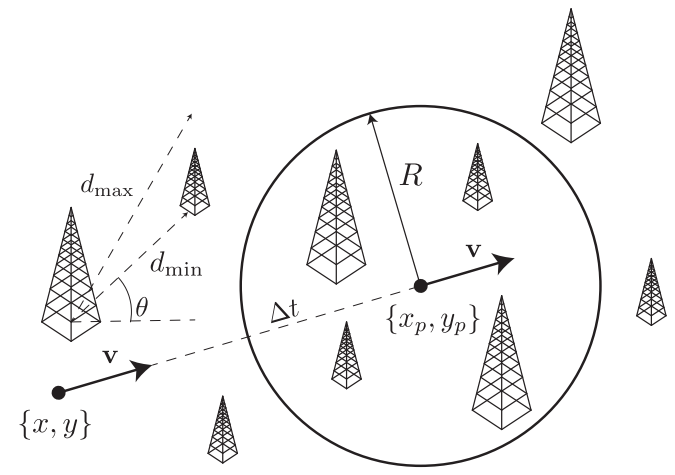

Fig. 1. Analyzed scenario for proposed REM handover algorithm.

accelerated or not linear, provided that the timespan is not too long the trajectory can be approximated by a set of adjacent segments where each follows a uniform linear motion. The endpoint is then introduced in the corresponding REMs to get an expected RSS value for a given cell. In order to reduce the number of queries, there is another algorithmrelated parameter, $R$, which basically defines a searching radius whose center is the predicted position. An example of this predicted position and the searching radius is depicted in Fig. 1. This parameter allows us to set an upper bound on the number of cells to be considered in the prediction calculations.

At this stage, despite the fact that we could just pick the strongest predicted cell in $\Delta t$, it is preferable to obtain a more unbiased measurement like the SINR. Besides, the expected QoS of the user will depend on the capacity of the channel rather than just the greatest received power. Taking all the previous parameters into account, our proposed policy to determine the strongest cell for handover can be expressed as

$$
c^{*}=\underset{c \in \mathcal{B}}{\operatorname{argmax}} \gamma_{c, u}(t+\Delta t) \mid \operatorname{RSS}_{s, u}^{\mathrm{TTT}}<\operatorname{RSS}_{c, u}^{\mathrm{TTT}}-\mathrm{HHM}
$$

where all parameters from (2) remain, and $c \in \mathcal{B}$ means cells within the searching radius $(\mathcal{B} \subset \mathcal{C})$. What this policy accomplishes is that a user will hand over to the strongest cell only if that same cell will have the highest SINR in the near future according to the user's trajectory. Note that (1) is a pessimistic evaluation of the SINR since the cell traffic load is not considered (all cells are fully loaded) and hence we could avoid some handovers towards heavily-loaded cells. This effect might increase the spectral efficiency and the global performance but this approach is not the actual focus of this work.

For instance, we can imagine a high-speed user crossing an area under the coverage of a macrocell and several femtocells. Even when the strongest-cell policy might suggest that the best option is handing over to a femtocell, after several seconds the same user will be handing over back to the macrocell. In our approach, we avoid such handovers, remaining camped on the macrocell. If we are dealing with a slowly-moving user, the current strongest cell will most likely have the best SINR in the future since the user will not have left its coverage. Needless to say, this introduces the second condition so as to perform a handover that, eventually, will lead to a reduction in the total number of handovers. The different steps of the proposed algorithm are shown in Algorithm 1.

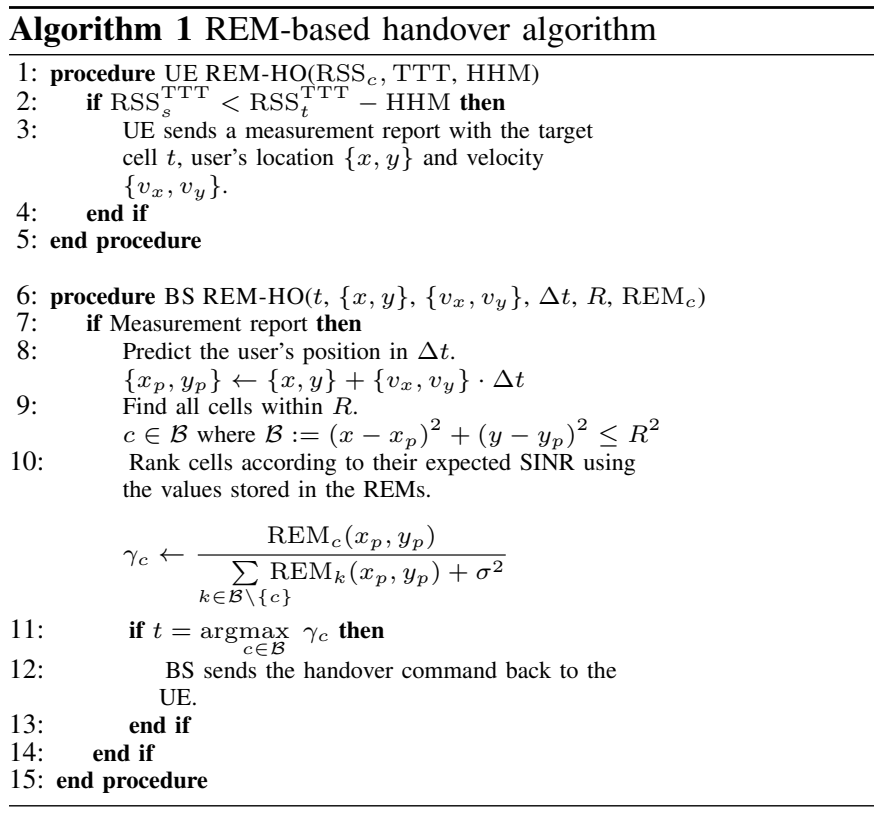

The procedure of the UE REM-HO takes place at the User Equiment and it is identical to what is currently used in the LTE-A standard. Similarly, the second procedure labeled BS REM-HO is run on the operator's side. On the user's side, the values used to trigger the events are not the raw physicallayer measurements but a filtered version using what is known as L3 filtering [9]. This recursive filter is located in the Radio Resource Control (RRC) layer and is expressed as

$$
F_{n}=(1-a) F_{n-1}+a M_{n}
$$

where $a=2^{-f c / 4}$ is the filter coefficient with parameter $f c$, $F_{n}$ is the filtered value (e.g. RSRP, RSRQ, etc.) and $M_{n}$ is the raw measurement provided by the PHY layer. In our case, $F_{n}$ is the RSS while the filter coefficient is optionally signaled by the network (the default value is $f c=4 \rightarrow a=0.5$ ). In a similar manner, the RSS values stored in the REMs only represent the effect of path loss and shadowing, since fastfading is averaged out.

\section{NUMERICAL RESUlTS}

\section{A. Simulation setup}

This section provides numerical results for evaluating the performance of the proposed REM-HO algorithm and, for comparison, the standard LTE-HO algorithm in a HetNet. The simulation environment used for carrying out the simulations has been developed entirely using MATLAB. It comprises a scenario generator based on hexagonal grids [10] for deploying base stations and users, a radio simulator following 
WINNER's Phase II Model implementation but using 3GPP 3D channel models [11] (see values in Table I) where we obtain the RSS for each pair BS-UE and, finally, an algorithm workbench where we can test different $\mathrm{HO}$ algorithms on the same radio simulation instance. The baseline scenario is based on the European-funded METIS-II project Performance Evaluation Framework User Case 1: Dense urban information society [12] that also includes mobility models. We have also included geographical distance-based wrapping following the 3GPP guidelines in [13]. Therefore, we do not have less interference at the border of the network. Table I lists the major simulation parameters. Macrocells, the first-tier network, follow the classic 19-site layout with an Inter-Site Distance (ISD) of $200 \mathrm{~m}$, while picocells, the second-tier network, are deployed following the next Probability Density Functions (PDF) using polar coordinates $(r, \theta)$ relative to their macro station [14]:

$$
\begin{array}{llrl}
p_{r}(r) & =\frac{2\left(r-d_{\min }\right)}{\left(d_{\max }-d_{\min }\right)^{2}} & d_{\min } & \leq r \leq d_{\max } \\
p_{\theta}(\theta) & =\frac{1}{2 \pi} & 0 & \leq \theta \leq 2 \pi
\end{array}
$$

where $d_{\min }$ is the closest distance admissible between tiers, which is equal to $55 \mathrm{~m}$, and $d_{\max }$ corresponds to the cell edge which depends on the angle $\theta$ (see details in Fig. 1). The UEs are also scattered according to (5) and (6) but changing the minimum distance to $35 \mathrm{~m}$ instead of $55 \mathrm{~m}$ [12]. For the baseline scenario, we have deployed 8 picocells and 5 users per macro sector unless otherwise stated. Each UE moves linearly in a direction randomly chosen between $[0,2 \pi)$, and its speed of motion is constant. During the simulation, the UE's position is updated every $100 \mathrm{~ms}$, and we obtain a channel realization every $10 \mathrm{~ms}$, which corresponds to the LTE-A frame duration. The propagation conditions (Line- and Non-Line-Of-Sight, LOS/NLOS) are assigned at the beginning according to the 3GPP formulae [11] and kept fixed for the rest of the simulation.

Similarly, the LTE-HO algorithm has been extracted from [2], including realistic timing such as the intra-frequency measurement period, handover preparation and execution times, etc. The RLF recovery has been bounded to occur within $120 \mathrm{~ms}$, including the RRC procedure delay. In this case, we assume that the target cell is sufficiently strong to be detected in the first correlation attempt [15]. A UE is said to be out of synchronization when its wideband SINR is below the threshold $Q_{\text {out }}$ and to be back in synchronization when above the threshold $Q_{\text {in }}$. An RLF is declared when a UE is out of synchronization for longer than the RLF Time (T310 timer in 3GPP terms, typically $1 \mathrm{~s}$ ). Subsequently, a handover failure happens if: a) RLF occurs before TTT duration, b) T310 is running when the handover command is sent, or c) the wideband SINR in the target cell is below $Q_{\text {out }}$ when the handover-complete message is sent. Finally, the Time of Stay (ToS) is defined as the duration between two consecutive successful handovers. In the event that these
TABLE I

\begin{tabular}{|c|c|c|}
\hline Items & Macrocell & Picocell \\
\hline Path-loss model & $\begin{array}{l}\text { 3GPP TR } 36.873 \\
\text { (3D-UMa) }[11]\end{array}$ & $\begin{array}{l}\text { 3GPP TR } 36.873 \\
\text { (3D-UMi) [11] }\end{array}$ \\
\hline Shadow fading std & $\begin{array}{l}\text { LOS }=4 \mathrm{~dB} \\
\text { NLOS }=6 \mathrm{~dB}\end{array}$ & $\begin{array}{l}\text { LOS }=3 \mathrm{~dB} \\
\text { NLOS }=4 \mathrm{~dB}\end{array}$ \\
\hline Correlation distance SF & $\begin{array}{l}\text { LOS }=37 \mathrm{~m} \\
\text { NLOS }=50 \mathrm{~m}\end{array}$ & $\begin{array}{l}\text { LOS }=10 \mathrm{~m} \\
\text { NLOS }=13 \mathrm{~m}\end{array}$ \\
\hline Fast-fading model & \multicolumn{2}{|c|}{ Geometry-based stochastic modeled [11] } \\
\hline BS antenna height & $25 \mathrm{~m}$ & $10 \mathrm{~m}$ \\
\hline BS/UE antenna pattern & \multicolumn{2}{|c|}{ Omnidirectional } \\
\hline BS EIRP & $49 \mathrm{dBm}$ & $30 \mathrm{dBm}$ \\
\hline Carrier frequency/BW & \multicolumn{2}{|c|}{$2 \mathrm{GHz} / 20 \mathrm{MHz}$} \\
\hline Inter-site distance & $200 \mathrm{~m}$ & $20 \mathrm{~m}$ \\
\hline UE height & \multicolumn{2}{|c|}{$1.5 \mathrm{~m}$} \\
\hline Sampling period & \multicolumn{2}{|c|}{$10 \mathrm{~ms}$} \\
\hline Total simulation time & \multicolumn{2}{|c|}{$300 \mathrm{~s}$} \\
\hline
\end{tabular}

SIMULATION PARAMETERS

TABLE II

HO AND RLF PARAMETERS

\begin{tabular}{l|l}
\hline Item & Description \\
\hline \hline$Q_{\text {out }}$ & $-8 \mathrm{~dB}$ \\
$Q_{\text {in }}$ & $-6 \mathrm{~dB}$ \\
RLF Time & $1 \mathrm{~s}$ \\
RLF Recovery Time & $120 \mathrm{~ms}$ \\
HO Preparation Time & $40 \mathrm{~ms}$ \\
HO Execution Time & $50 \mathrm{~ms}$ \\
Minimum ToS & $1 \mathrm{~s}$ \\
\hline
\end{tabular}

handovers are performed between the same two cells in less than the minimum ToS, it is considered a ping-pong handover. Ping-pong handovers reduce the efficiency of the network so it is crucial to reduce their numbers while keeping a satisfactory QoS. Table II reflects the parameters involved in both handover and RLF.

REMs have been built using the worst-case scenario for both macro and picocells, in effect, NLOS, where path loss and large-scale fading can be predicted but small-scale fading cannot due to its nature. If we were able to build REMs from real measurements, this workaround would not be needed since the measurements themselves inherently contain the propagation conditions. Finally, we assess the proposed algorithm and its counterpart by evaluating 4 different sets of configuration parameters as shown in Table III, ranging from less to more aggressive (lower to higher mobility as well), extracted from [2]. We have decided to keep the specific REM-HO parameters constant on purpose so that we can evaluate them later on. $R$ in Algorithm 1 is $200 \mathrm{~m}$ to coincide with the ISD and $\Delta t$ is $2.5 \mathrm{~s}$, which is a compromise for the different speeds considered $(3,30,60,120 \mathrm{~km} / \mathrm{h})$.

\section{B. Ping-pong handovers}

Table IV shows the ping-pong rate percentage defined in [2] as

Ping-pong rate $(\%)=\frac{\text { number of ping-pongs }}{\text { total number of successful handovers }} \cdot 100$. 
TABLE III

CONFIGURATION SETS

\begin{tabular}{lllll}
\hline Profile & Set 1 & Set 2 & Set 3 & Set 4 \\
\hline \hline TTT [ms] & 160 & 160 & 80 & 40 \\
HHM [dB] & 3 & 2 & 1 & -1 \\
\hline
\end{tabular}

TABLE IV

AVERAGE PING-PONG RATE (\%) WITH 8 PICOCELLS PER MACROCELL SECTOR AND $\Delta t=2.5 \mathrm{~S}$

\begin{tabular}{clllll}
\hline & Speed $[\mathbf{k m} / \mathbf{h}]$ & Set 1 & Set 2 & Set 3 & Set 4 \\
\hline \hline \multirow{4}{*}{ LTE-HO } & 3 & 0 & 1.6721 & 19.8882 & 58.6097 \\
& 30 & 2.1754 & 4.0750 & 8.7126 & 25.5029 \\
& 60 & 4.2724 & 7.3409 & 11.8169 & 21.6473 \\
& 120 & 3.8307 & 6.9485 & 18.1408 & 26.0302 \\
\hline \multirow{2}{*}{ REM-HO } & 3 & 0 & 0 & 0.4562 & 1.9008 \\
& 30 & 0.0867 & 0.1725 & 0.4497 & 1.2736 \\
& 120 & 0 & 0 & 0.1077 & 0.2356 \\
REM-HO & 3 & 0 & 0 & 0.0249 & 0.0598 \\
with 3 & 30 & 0 & 1.5751 & 19.0726 & 56.3074 \\
candidates & 60 & 1.0745 & 2.2814 & 5.4542 & 16.0515 \\
& 120 & 0.2964 & 1.8212 & 3.9878 & 9.4934 \\
\hline
\end{tabular}

It can be seen that in our proposed algorithm this rate is below 2 percent in any configuration set regardless of the user's speed. The reason behind such a considerable reduction is the incomplete channel states stored in the REMs. Our technique double-checks if a target cell spotted by a user would still be a good choice in $\Delta t$ seconds, so avoiding virtually any possibility of hopping over two cells in less than $\Delta t$. The users with the lowest speed suffer from a marginally higher rate because they require more time to cross the same area. An adaptive timespan could be considered according to the user's speed, enunciating an optimization problem.

If we relax the constraint of picking the BS with the highest SINR and we increase the candidate list up to the first $K$ cells with the highest SINR, our proposed technique will tend to behave like LTE-A. As $K$ increases, both algorithms become indistinguishable. For example, in Table IV we have set $K$ equal to 3. Again, the lowest-speed users are the most affected by this change, with rates similar to LTE-A for configuration sets 3 and 4 (see the set details in Table III). The rest of the sets do not increase at the same pace due to the longer TTT.

The decrease in the ping-pong rate has a clear impact on the number of handovers. Table $\mathrm{V}$ shows the average number of events where REM-HO events are substantially diminished. The gray rows represent the ratio of ping-pong handovers to the total. The difference in the number of handovers is not only attributed to the ping-pong avoidance; in this regard, our algorithm postpones handovers as long as possible until a strong cell which will stay strong for at least $\Delta t$ seconds shows up. This effect is translated into a higher occurrence of RLFs but is still comparable to LTE-A for the most aggressive sets as shown in Table VI. There is a trade-off between the ping-pong rate and the number of RLFs, controlled by the size of the candidate list. As previously mentioned, being less
TABLE V

AVERAGE NUMBER OF HANDOVERS PER USER PER SECOND (HO/UE/S) WITH 8 PICOCELLS PER MACROCELL SECTOR AND $\Delta t=2.5 \mathrm{~S}$

\begin{tabular}{|c|c|c|c|c|c|}
\hline & Speed $[\mathrm{km} / \mathrm{h}]$ & Set 1 & Set 2 & Set 3 & Set 4 \\
\hline \multirow{8}{*}{ LTE-HO } & \multirow{2}{*}{3} & 0.0014 & 0.0024 & 0.0056 & 0.0190 \\
\hline & & 0 & 0 & 0.0011 & 0.0111 \\
\hline & \multirow{2}{*}{30} & 0.0034 & 0.0058 & 0.0130 & 0.0213 \\
\hline & & 0.0001 & 0.0002 & 0.0011 & 0.0054 \\
\hline & \multirow{2}{*}{60} & 0.0031 & 0.0053 & 0.0168 & 0.0260 \\
\hline & & 0.0001 & 0.0004 & 0.0020 & 0.0056 \\
\hline & \multirow{2}{*}{120} & 0.0031 & 0.0050 & 0.0171 & 0.0266 \\
\hline & & 0.0001 & 0.0003 & 0.0031 & 0.0069 \\
\hline \multirow{8}{*}{$\begin{array}{l}\text { REM-HO } \\
(\text { gray rows } \\
1 \mathrm{E}-4 \times)\end{array}$} & \multirow{2}{*}{3} & 0.0010 & 0.0013 & 0.0019 & 0.0024 \\
\hline & & 0 & 0 & 0.0858 & 0.4600 \\
\hline & \multirow{2}{*}{30} & 0.0009 & 0.0014 & 0.0028 & 0.0034 \\
\hline & & 0.0078 & 0.0234 & 0.1248 & 0.4366 \\
\hline & \multirow{2}{*}{60} & 0.0005 & 0.0008 & 0.0022 & 0.0026 \\
\hline & & 0 & 0 & 0.0234 & 0.0624 \\
\hline & \multirow{2}{*}{120} & 0.0005 & 0.0009 & 0.0031 & 0.0039 \\
\hline & & 0 & 0 & 0.0078 & 0.0234 \\
\hline
\end{tabular}

TABLE VI

AVERAGE NUMBER OF RLFS PER USER PER SECOND (RLF/UE/S) WITH 8 PICOCELLS PER MACROCELL SECTOR AND $\Delta t=2.5 \mathrm{~S}$

\begin{tabular}{llllll}
\hline & Speed $[\mathbf{k m} / \mathbf{h}]$ & Set 1 & Set 2 & Set 3 & Set 4 \\
\hline \hline \multirow{4}{*}{ LTE-HO } & 3 & 0.0027 & 0.0024 & 0.0020 & 0.0018 \\
& 30 & 0.0090 & 0.0082 & 0.0042 & 0.0033 \\
& 120 & 0.0145 & 0.0142 & 0.0079 & 0.0061 \\
\multirow{5}{*}{ REM-HO } & 3 & 0.0177 & 0.0176 & 0.0117 & 0.0099 \\
\hline & 60 & 0.0024 & 0.0022 & 0.0020 & 0.0020 \\
& 120 & 0.0080 & 0.0076 & 0.0063 & 0.0059 \\
& & 0.0122 & 0.0119 & 0.0106 & 0.0102 \\
& & 0.0148 & 0.0144 & 0.0123 & 0.0116 \\
\hline
\end{tabular}

conservative and allowing more cells to be selected can reduce the RLFs while increasing the ping-pongs.

\section{Impact on spectral efficiency}

Preventing users from handing over also brings a loss in a user's spectral efficiency, as in Fig. 2 and Fig. 3. In these figures we have the average channel spectral efficiency for configuration set 3 (TTT $=80 \mathrm{~ms}, \mathrm{HHM}=1 \mathrm{~dB}$ ) for $60 \mathrm{~km} / \mathrm{h}$ users. In Fig. 2 we keep all the parameters fixed and sweep only $\Delta t$. The obtained loss in spectral efficiency compared to LTE-A can be easily explained. LTE-A picks the strongest cell at any time after TTT seconds whereas we double-check if that cell will maintain its status after $\Delta t$ seconds. Therefore, if the timespan becomes smaller, the filtered measurements and the predicted values stored in the REMs will be similar because of the spatial correlation in large-scale fading. For instance, our algorithm almost perfectly overlaps LTE-A when $\Delta t=0$. Selecting the right $\Delta t$ for each speed is key to control the trade-off between the reduction in the ping-pong handovers and the loss in spectral efficiency. As an example, for $\Delta t=$ $2.5 \mathrm{~s}$, which corresponds to a prediction distance of $41.67 \mathrm{~m}$, we reduce the ping-pong rate to $0.01 \%$ ( $11.82 \%$ for LTE-HO) but, at the same time, we loss approximately $9 \%$ of spectral efficiency $(1.14 \mathrm{~b} / \mathrm{s} / \mathrm{Hz}$ against $1.25 \mathrm{~b} / \mathrm{s} / \mathrm{Hz})$. 


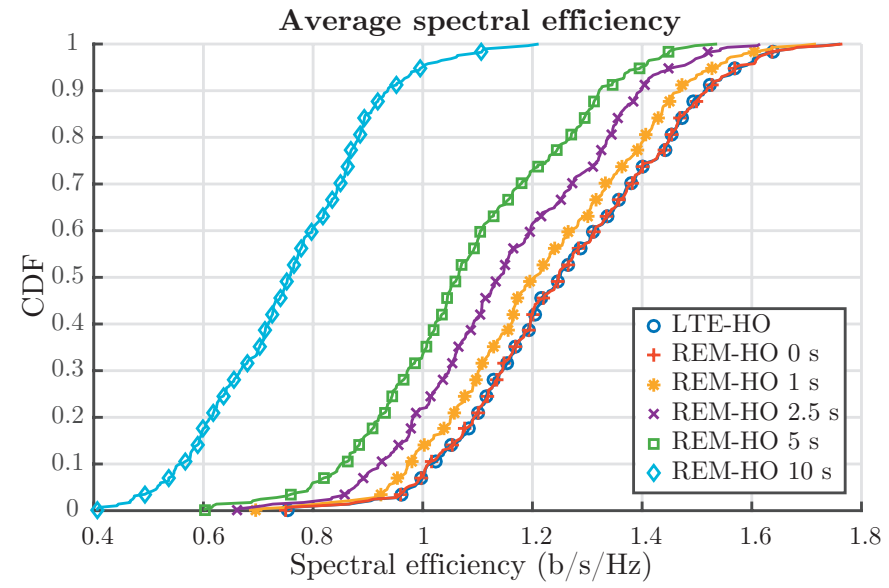

Fig. 2. CDF of channel spectral efficiency for configuration set $3,60 \mathrm{~km} / \mathrm{h}$, 8 picocells and $\Delta t=\{1,2.5,5,10\} \mathrm{s}$.

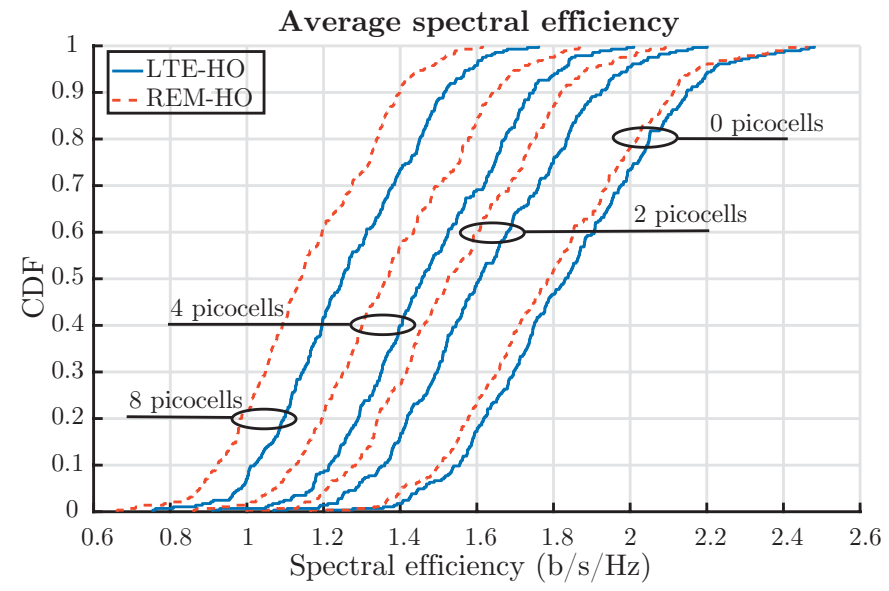

Fig. 3. CDF of channel spectral efficiency for configuration set $3,60 \mathrm{~km} / \mathrm{h}$, $\Delta t=2.5 \mathrm{~s}$ and $\{0,2,4,8\}$ picocells.

In Fig. 3 we benchmark the performance of the LTE$\mathrm{HO}$ and the proposed REM-HO algorithms under different picocell densities. Although the gap increases with density, it will not go further than approximately $0.1 \mathrm{~b} / \mathrm{s} / \mathrm{Hz}$. Here we observe the trade-off of using our technique instead of the current LTE-A handover approach. The slight loss of spectral efficiency is due to the smaller overall number of handovers, by around an order of magnitude. However, we ensure an acceptable QoS while introducing a smaller amount of overhead in the network. Regarding the network overhead, the UEs only send a few extra bits of information compared to the LTE-HO algorithm (location and velocity although they are parameters already included in the standard), that we might consider negligible. Besides, the signaling between BSs will depend on the specific algorithm, as detailed in [4], it is estimated that the REM signaling requirement is about $68 \mathrm{bits} / \mathrm{s}$ per cell for an inter-cell interference minimization technique. Yet the update frequency of the databases is not discussed.

\section{CONCLUSION}

In this work, we have introduced the idea of using REMs in the handover decision-making as a way of predicting QoS. We have also presented a handover algorithm which takes advantage of REMs' spatial information in multi-tier cellular networks by preventing users from handing over too early. We have simulated our proposed algorithm and the LTE$\mathrm{HO}$ algorithm where we have shown a significant reduction in the number of ping-pong handovers independently of the hysteresis margin applied. At the same time, the overall number of handovers is also cut down while maintaining a reasonable channel spectral efficiency.

\section{ACKNOWLEDGMENT}

This paper has been funded by a Cotutelle International Macquarie University Research Excellence Scholarship (iMQRES) - No. 2016188.

\section{REFERENCES}

[1] "White paper: Cisco VNI Forecast and Methodology, 2016-2021," https://www.cisco.com/c/en/us/solutions/collateral/service-provider/ visual-networking-index-vni/complete-white-paper-c11-481360.html, last accessed in September 2017.

[2] $3^{\text {rd }}$ Generation Partnership Project, "Evolved Universal Terrestrial Radio Access (E-UTRA); Mobility enhancements in heterogeneous networks," 3GPP TR 36.839 V11.1.0, Tech. Rep., 2012.

[3] D. Xenakis, N. Passas, L. Merakos, and C. Verikoukis, "Mobility Management for Femtocells in LTE-Advanced: Key Aspects and Survey of Handover Decision Algorithms," IEEE Communications Surveys and Tutorials, vol. 16, no. 1, pp. 64-91, 2014.

[4] J. Perez-Romero, A. Zalonis, L. Boukhatem, A. Kliks, K. Koutlia, N. Dimitriou, and R. Kurda, "On the use of radio environment maps for interference management in heterogeneous networks," IEEE Communications Magazine, vol. 53, no. 8, pp. 184-191, August 2015.

[5] B. A. Jayawickrama, E. Dutkiewicz, I. Oppermann, G. Fang, and J. Ding, "Improved performance of spectrum cartography based on compressive sensing in cognitive radio networks," in 2013 IEEE International Conference on Communications (ICC), Conference Proceedings, pp. $5657-5661$

[6] F. Letourneux, Y. Corre, E. Suteau, and Y. Lostanlen, "3D coverage analysis of LTE urban heterogeneous networks with dense femtocell deployments," EURASIP Journal on Wireless Communications and Networking, vol. 2012, no. 1, p. 319, Oct 2012. [Online]. Available: https://doi.org/10.1186/1687-1499-2012-319

[7] $3^{\text {rd }}$ Generation Partnership Project, "Evolved Universal Terrestrial Radio Access (E-UTRA); Requirements for support of radio resource management," 3GPP TR 36.133 V14.0.0, Tech. Rep., 2016.

[8] J. Johansson, W. A. Hapsari, S. Kelley, and G. Bodog, "Minimization of drive tests in 3GPP release 11," IEEE Communications Magazine, vol. 50, no. 11, pp. 36-43, 2012.

[9] H. Holma, A. Toskala, and J. Reunanen, LTE Small Cell Optimization: 3GPP Evolution to Release 13. ISBN: 978-1-118-91257-7: John Wiley \& Sons, 2015.

[10] "Hexagonal Grids from Red Blob Games," http://www.redblobgames. com/grids/hexagons/, last accessed in September 2017.

[11] $3^{\text {rd }}$ Generation Partnership Project, "Study on 3D channel model for LTE,” 3GPP TR 36.873 V12.2.0, Tech. Rep., 2015.

[12] Mobile and wireless communications Enablers for Twenty-twenty (2020) Information Society, "Performance evaluation framework," METIS-II Deliverable D2.1, Tech. Rep., 2016.

[13] $3^{\text {rd }}$ Generation Partnership Project, "Discussions on Wrapping Methodology," 3GPP TSG RAN WG1 Meeting \#76 R1-140842, Tech. Rep., 2014.

[14] M. S. Alouini and A. J. Goldsmith, "Area spectral efficiency of cellular mobile radio systems," IEEE Transactions on Vehicular Technology, vol. 48, no. 4, pp. 1047-1066, 1999.

[15] S. Sesia, I. Toufik, and M. Baker, LTE - The UMTS Long Term Evolution: From Theory to Practice, 2nd Edition. ISBN: 978-0-47066025-6: John Wiley \& Sons, 2011. 